\title{
Palliative chemotherapy with or without anti-EGFR therapy for de novo metastatic nasopharyngeal carcinoma: a propensity score-matching study
}

\author{
This article was published in the following Dove Press journal:
} Drug Design, Development and Therapy

\author{
Xue-Song Sun ${ }^{1-3, *}$ \\ Yu-Jing Liang ${ }^{1-3, *}$ \\ Xiao-Yun $\mathrm{Li}^{1-3, *}$ \\ Sai-Lan Liu ${ }^{1-3}$ \\ Qiu-Yan Chen ${ }^{1-3}$ \\ Lin-Quan Tang ${ }^{1-3}$ \\ Hai-Qiang Mai ${ }^{1-3}$
}

'Department of Nasopharyngeal Carcinoma, Sun Yat-sen University Cancer Centre, Guangzhou, People's Republic of China; ${ }^{2}$ Department of Nasopharyngeal Carcinoma, State Key Laboratory of Oncology in South China, Guangzhou, People's Republic of China;

${ }^{3}$ Department of Nasopharyngeal Carcinoma, Collaborative Innovation Centre for Cancer Medicine, Guangzhou, People's Republic of China

*These authors contributed equally to this work

\begin{abstract}
Objective: We aimed to investigate the efficacy and safety of cetuximab (CTX) or nimotuzumab (NTZ) on the addition of palliative chemotherapy (PCT) in patients with de novo metastatic nasopharyngeal carcinoma (NPC).

Materials and methods: From 2007 to 2016, 451 eligible patients with de novo metastatic NPC were enrolled in the study. With propensity score matching technique, we created a well-balanced cohort by matching patients who received CTX/NTZ plus PCT (62 patients) with those receiving PCT alone (248 patients) in a ratio of 1:4. The primary endpoint was overall survival (OS). All potential prognostic factors were involved in the multivariate analysis with the Cox regression hazards model. Kaplan-Meier curves were used to compare the survival status, and log-rank test to measure the significance.

Results: The median follow-up time was 27.7 months (range, 1-126 months). No significant difference in survival was observed between the CTX/NTZ plus PCT group and PCT group. (3-year OS: $63.0 \%$ vs $58.1 \% ; P=0.485$ ). The administration of CTX/NTZ was not found to be an independent prognostic factor in multivariate analysis. With regard to toxicity, the development of a G3-4 skin reaction and mucositis was more common in patients receiving CTX plus PCT. Interaction effects analysis did not show any significant interaction effects on OS between the treatment regimen and prognostic factors $(P>0.05)$.

Conclusion: The efficacy of CTX/NTZ and PCT is comparable to single PCT treatment in terms of survival outcomes among de novo metastatic NPC patients. Moreover, the application of CTX exacerbated skin reactions and mucositis.
\end{abstract}

Keywords: targeted drug, chemotherapy, treatment, nasopharyngeal carcinoma and overall survival

\section{Introduction}

Nasopharyngeal cancer (NPC) is a unique subtype in head and neck cancers both anatomically and biologically; it causes an estimated 51,000 deaths annually, which accounted for $0.6 \%$ of all cancer-related deaths worldwide in $2012 .{ }^{1}$ Due to the hypersensitivity of NPC to radiotherapy, the combination of radiotherapy with chemotherapy has become the cornerstone treatment for locoregional advanced NPC patients, which has been validated by high-level evidence that a satisfactory 5 -year survival rate of approximately $75 \%$ has been achieved. ${ }^{2,3}$ However, advanced NPC patients are prone to develop distant metastasis, ${ }^{4}$ and approximately $15 \%$ of NPC patients are detected with metastatic lesions at the time of initial diagnosis. ${ }^{5}$ The overall survival (OS) of metastatic NPC patients is poor, and the
Correspondence: Lin-Quan Tang; Hai-Qiang Mai

Department of Nasopharyngeal Carcinoma, Sun Yat-sen University Cancer Center, 65I Dongfeng Road East, Guangzhou 510060, People's

Republic of China

Tel +86 2087343643

Fax +862087343392

Email maihq@mail.sysu.edu.cn; tanglq@sysucc.org.cn 
median OS reported following first-line chemotherapy is reportedly 29.1 months, which presents crucial challenges for the treatment of metastatic NPC. ${ }^{6}$

Epidermal growth factor receptor (EGFR), also known as ErbB1, has been considered as an important therapeutic target for NPC as increasing evidence indicated that EGFR signaling plays a vital role in NPC pathogenesis. ${ }^{7}$ EGFR is reportedly overexpressed in $80-89 \%$ of NPC patients, which may be responsible for treatment resistance and poor prognosis. ${ }^{4,8}$ Cetuximab (CTX), a chimeric (mouse/ human) monoclonal antibody is the first EGFR inhibitor studied clinically in NPC, and has shown effectiveness in recurrent or metastatic NPC patients. ${ }^{7,9}$ The humanized therapeutic monoclonal antibody nimotuzumab (NTZ) has also been applied in locoregional advanced NPC. Satisfactory efficacy and tolerable side effects in comparison with chemotherapy have been reported. ${ }^{10-12}$ Nevertheless, data on initially metastatic NPC patients treated with PCT in combination with or without NTZ/ CTX has been poorly documented. The influence of EGFR monoclonal antibody in this group remains largely unknown. Therefore, in the present study, we aimed to identify the effect of CTX or NTZ in de novo metastatic NPC patients, and provide further information for the treatment of metastatic NPC patients.

\section{Materials and methods}

\section{Patient population}

From 2007 to 2016, 451 de novo metastatic NPC patients were enrolled in our retrospective cohort analysis. The inclusion criteria were as follows: (1) pathologically confirmed NPC; (2) received cisplatin-based palliative chemotherapy (PCT) (3) initial Karnofsky performance score (KPS) >70; (4) normal organ functions; (5) no pregnancy, lactation, or second malignant disease. Using propensity scores adjusted for age, gender, $\mathrm{T}$ stage, $\mathrm{N}$ stage, metastatic sites, PCT cycles, and the use of locoregional radiotherapy (LRRT), a well-balanced cohort was created, wherein each patient receiving CTX/NTZ plus PCT was matched with 4 patients receiving PCT alone. The flow chart was shown in Figure 1. Our study was approved by the clinical research ethics committee of SYSUCC.

\section{Diagnosis and treatment}

Before diagnosis, patients underwent a series of evaluations, including physical examination, nasopharyngoscopy and pathology assessment, magnetic resonance imaging
(MRI)/computed tomography (CT) with contrast for head and neck and metastatic lesions, chest radiography/CT with contrast, abdominal ultrasound/CT with contrast, and bone scan for whole-body assessment or positron emission tomography-computed tomography (PET/CT) as a substitute.

Platinum-based palliative chemotherapy with or without CTX/NTZ was administered in all patients in this study. The common chemotherapy regimens were as follows-TP: docetaxel $\left(80 \mathrm{mg} / \mathrm{m}^{2} \mathrm{~d} 1\right)$ plus cisplatin $(75 \mathrm{mg}$ / $\left.\mathrm{m}^{2} \mathrm{~d} 1\right)$, PF: cisplatin $\left(20-25 \mathrm{mg} / \mathrm{m}^{2} \mathrm{~d} 1-3\right)$ plus 5-fluorouracil $\left(800-1000 \mathrm{mg} / \mathrm{m}^{2}, 120 \mathrm{~h}\right)$, TPF: docetaxel $\left(60 \mathrm{mg} / \mathrm{m}^{2}\right.$ d1) plus cisplatin $\left(60 \mathrm{mg} / \mathrm{m}^{2} \mathrm{~d} 1\right)$ plus 5 -fluorouracil (500$800 \mathrm{mg} / \mathrm{m} 2,120 \mathrm{~h})$, and GP: gemcitabine $\left(1000 \mathrm{mg} / \mathrm{m}^{2}\right.$ $\mathrm{d} 1,8)$ combined with cisplatin (20-30 $\left.\mathrm{mg} / \mathrm{m}^{2} \mathrm{~d} 1-3\right)$. Chemotherapy was intravenously administered at 3-week intervals and the median cycle of PCT was five (range: 210 cycles). CTX was administered at an initial dose of $400 \mathrm{mg} / \mathrm{m}^{2}$, followed by a weekly dose of $250 \mathrm{mg} / \mathrm{m}^{2}$, whereas NTZ was intravenously administered at a median dose of $200 \mathrm{mg}$ weekly. The EGFR-targeted drugs were applied combined with PCT. Forty-nine patients in CTX/ NTZ+PCT group received LRRT after PCT and 21 patients received EGFR-targeted drugs during radiotherapy.

\section{Follow-up and outcome}

Assessments were performed in patients at 3-month intervals during the first 3 years, and at 6-month intervals thereafter until death. Physical examination, nasopharyngoscopy, MRI/CT with contrast of head and neck metastatic lesions, chest radiography/CT with contrast, and abdominal ultrasound/CT with contrast were routinely performed during follow up. PET/CT and other examinations would be considered when necessary. The primary endpoint in this study was OS, which was defined as the time from the date of diagnosis to the date of death from any cause.

\section{Statistical analysis}

In this study, continuous variables were converted into categorical variables based on the clinical application or median regular cut-off points. A multivariate logistic regression model was used to calculate the propensity score for each patient, based on the following covariates: gender, age, radiation therapy, tumor stage, lymph node stage, PCT cycles, and metastatic site. Moreover, a 1:4 matching protocol without replacement (greedy matching algorithm) was used, and the caliper width was equal to 


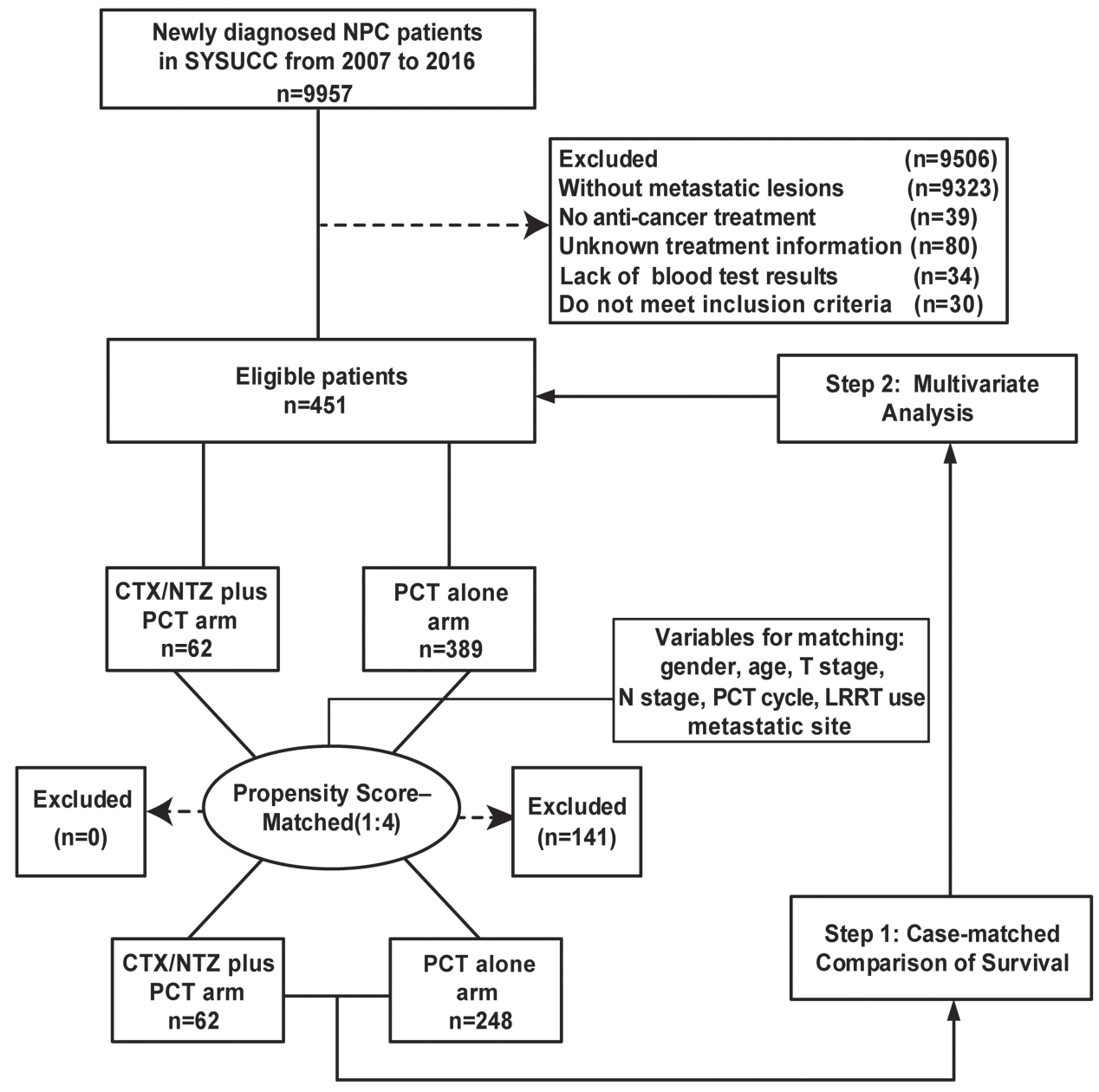

Figure I Flow chart of patient inclusion.

0.05 of the logit standard deviation of the propensity score. Kaplan-Meier survival curves were used to compare patient survival with the log-rank test. A Cox proportional hazard model was used to perform multivariate analyses to estimate the hazard ratios (HRs) and 95\% confidence intervals (CIs) for the relationship between the characteristics and OS. The Enter method was used for multivariable Cox regression. Statistical analyses were performed using the Statistical Package for Social Sciences 24.0 (IBM Corporation, Armonk, NY, USA) and R (http:// www.R-project.org). Statistical tests in this study were two-tailed and $P<0.05$ was considered to indicate statistical significance.

\section{Result}

\section{Patient characteristics}

From 2007 to 2016, 451 de novo metastatic NPC patients were enrolled in our retrospective study. In order to reduce the potential confounding, we created a well-balanced cohort using propensity score matching (PSM) with a 1:4 ratio, including 62 patients in the CTX/NTZ plus PCT group and 248 patients in the PCT alone group. Among these patients, the male-to-female ratio was 5.1:1 and the average age of all patients was 46 years. In patients treated with an anti-EGFR drug, NTZ was more frequently used (37/62, 59.7\%), in comparison with CTX (25/62, 40.3\%). Other patient characteristics are shown in Table 1.

\section{Survival analysis}

Of the 451 patients, $224(49.7 \%)$ died with a median follow-up of 27.7 months (range, 1-126 months). The 1-, 3 -, and 5-year OS rates were $90.4 \%, 56.3 \%$ and $39.9 \%$, respectively. We compared the survival status based on the treatment method in PSM cohort, and found that the 3-year OS was similar in patients who received NTZ/CTX, in comparison with patients who did not $(63.0 \%$ vs $58.1 \%$; 
Table I Clinical characteristic in entire cohort and PSM cohort

\begin{tabular}{|c|c|c|c|c|c|c|}
\hline \multirow[b]{2}{*}{ Characteristic } & \multicolumn{3}{|c|}{$\begin{array}{l}\text { The entire cohort } \\
\text { CTX/NTZ plus PCT } \\
\text { P-value }\end{array}$} & \multicolumn{3}{|c|}{$\begin{array}{l}\text { Propensity score matching cohort } \\
\text { CTX/NTZ plus PCT } \\
\text { P-value }\end{array}$} \\
\hline & PCT alone & CTX/NTZ plus PCT & \multirow[t]{2}{*}{$P$-value } & PCT alone & CTX/NTZ plus PCT & \multirow[t]{2}{*}{$P$-value } \\
\hline Total & $N=389$ & $N=62$ & & $\mathrm{~N}=\mathbf{2 4 8}$ & $N=62$ & \\
\hline \multicolumn{7}{|l|}{ Gender } \\
\hline Male & $323(83.0 \%)$ & 52 (83.9\%) & 1.000 & 207 (83.5\%) & 52 (83.9\%) & 1.000 \\
\hline Female & $66(17.0 \%)$ & $10(16.1 \%)$ & & $4 \mathrm{l}(16.5 \%)$ & $10(16.1 \%)$ & \\
\hline \multicolumn{7}{|l|}{ Age (years) } \\
\hline$\leq 46$ & 190 (48.8\%) & $36(58.1 \%)$ & 0.218 & $13 \mid(52.8 \%)$ & $36(58.1 \%)$ & 0.480 \\
\hline$>46$ & $199(51.3 \%)$ & 26 (4I.9\%) & & 117 (47.2\%) & 26 (4I.9\%) & \\
\hline \multicolumn{7}{|l|}{ T stage s $^{\#}$} \\
\hline TI & 17 (4.4\%) & I (I.6\%) & 0.595 & $6(2.4 \%)$ & I (I.6\%) & 0.695 \\
\hline $\mathrm{T} 2$ & $52(13.4 \%)$ & $6(9.7 \%)$ & & 33 (13.3\%) & $6(9.7 \%)$ & \\
\hline T3 & 191 (49.1\%) & $33(53.2 \%)$ & & 112 (45.2\%) & $33(53.2 \%)$ & \\
\hline T4 & $129(33.2 \%)$ & $22(35.5 \%)$ & & 97 (39.1\%) & $22(35.5 \%)$ & \\
\hline \multicolumn{7}{|l|}{$N$ stage ${ }^{\#}$} \\
\hline No & $14(3.6 \%)$ & $0(0.0 \%)$ & 0.332 & $6(2.4 \%)$ & $0(0.0 \%)$ & $0.654 *$ \\
\hline $\mathrm{NI}$ & 72 (18.5\%) & 8 (I2.9\%) & & 27 (10.9\%) & $8(12.9 \%)$ & \\
\hline N2 & I55 (39.8\%) & $28(45.2 \%)$ & & $100(40.3 \%)$ & $28(45.2 \%)$ & \\
\hline N3 & 148 (38.0\%) & $26(41.9 \%)$ & & 115 (46.4\%) & $26(41.9 \%)$ & \\
\hline \multicolumn{7}{|l|}{ Metastatic sites } \\
\hline Bone & I 78 (45.8\%) & $29(46.8 \%)$ & 0.536 & $128(51.6 \%)$ & $29(46.8 \%)$ & 0.666 \\
\hline Lung & 51 (13.1\%) & 7 (II.3\%) & & 29 (11.7\%) & 7 (II.3\%) & \\
\hline Liver & 36 (9.3\%) & $9(14.5 \%)$ & & 21 (8.5\%) & 9 (14.5\%) & \\
\hline Distant nodes & $28(7.2 \%)$ & $6(9.7 \%)$ & & $20(8.1 \%)$ & $6(9.7 \%)$ & \\
\hline Multiple sites & 96 (24.7\%) & II (I7.7\%) & & $50(20.2 \%)$ & II (I7.7\%) & \\
\hline \multicolumn{7}{|l|}{ LRRT use } \\
\hline No & $130(33.4 \%)$ & I3 (2I.0\%) & 0.056 & 49 (19.8\%) & $13(2 \mid .0 \%)$ & 0.860 \\
\hline Yes & 259 (66.6\%) & 49 (79.0\%) & & 199 (80.2\%) & 49 (79.0\%) & \\
\hline \multicolumn{7}{|l|}{ PCT regimen } \\
\hline TPF & I0I (26.0\%) & 17 (27.4\%) & 0.095 & 69 (27.8\%) & 17 (27.4\%) & 0.105 \\
\hline $\mathrm{TP}$ & $82(21.1 \%)$ & 20 (32.3\%) & & 52 (21.0\%) & $20(32.3 \%)$ & \\
\hline $\mathrm{PF}$ & 107 (27.5\%) & 8 (I2.9\%) & & $64(25.8 \%)$ & 8 (I2.9\%) & \\
\hline GP & $23(5.9 \%)$ & $5(8.1 \%)$ & & II (4.4\%) & $5(8.1 \%)$ & \\
\hline Others & 76 (19.5\%) & 12 (19.4\%) & & $52(21.0 \%)$ & $12(19.4 \%)$ & \\
\hline \multicolumn{7}{|l|}{ PCT cycle } \\
\hline$\leq 4$ & I5I (38.8\%) & 21 (33.9\%) & 0.485 & 96 (38.7\%) & 21 (33.6\%) & 0.559 \\
\hline$>4$ & $238(61.2 \%)$ & 41 (66.1\%) & & 152 (61.3\%) & $41(66.1 \%)$ & \\
\hline
\end{tabular}

Notes: ${ }^{*}$ According to the 8 th edition of the UICC/AJCC staging system. *The P-value was calculated with the Pearson $\chi 2$ test or Fisher's exact test.

Abbreviations: PSM, Propensity score matching; NTZ, nimotuzumab; CTX, cetuximab; LRRT, locoregional radiotherapy; PCT, palliative chemotherapy; TPF, cisplatin plus docetaxel plus 5-fluorouracil; TP, cisplatin plus docetaxel; PF, cisplatin plus 5-fluorouracil; GP, cisplatin plus gemcitabine.

95\% CI: 49.3-76.7\% vs 52.6-63.6\%). There were no significant differences in the risk of death (HR, 0.86; 95\% CI, $0.56-1.31 ; P=0.485)$. The Kaplan-Meier curves are shown in Figure 2. Furthermore, we investigated whether the different EGFR drugs influenced the survival of these patients. As shown in Figure S1, there was no significant survival difference between patients treated with CTX and NTZ $(P=0.279)$. 


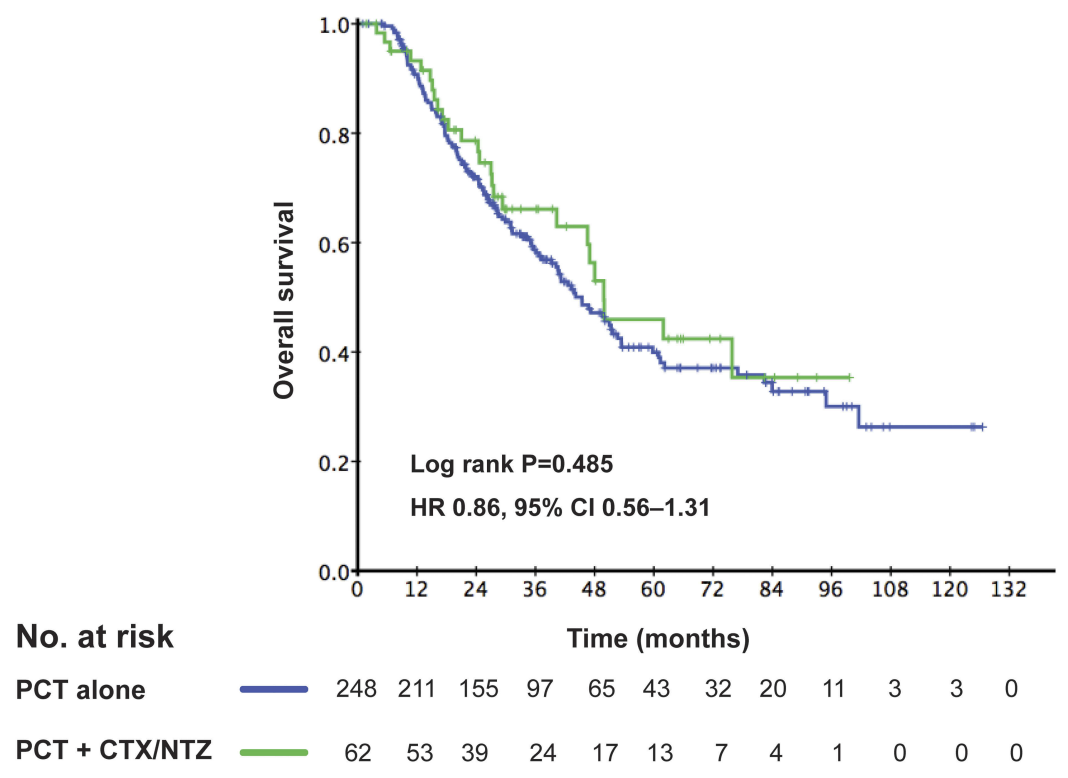

Figure 2 Kaplan-Meier OS curves in the well balanced cohort of patients receiving CTX/NTZ+PCT or PCT alone.

\section{Toxicity}

The effect of toxicity in each group in the PSM cohort was shown in Table 2. The rates of serious (G3-G4) hematological toxicities were similar between two groups. The incidence of G3 vomiting was in a low level and comparable between groups. However, a higher frequency of G3 skin reactions $(29.0 \%$ vs $6.9 \%, P<0.001)$ and G3-4 mucositis $(38.7 \%$ vs $10.9 \%, P<0.001)$ was noted in the CTX NTZ plus PCT group, relative to the PCT alone group. No significant differences among the treatment groups were observed in terms of hepatotoxicity and nephrotoxicity. In subgroup analysis, patients receiving CTX were significantly more inclined to suffer from G3-4 neutropenia ( $48 \%$ vs $31.4 \%, P=0.016)$, G3 skin reactions $(48.0 \%$ vs $16.2 \%, P=0.016)$ and $\mathrm{G} 3-4$ mucositis $(64.0 \%$ vs $21.6 \%$, $P=0.003$ ), as compared to those receiving NTZ, which manifested a less safer profile of CTX. Besides, the effect of toxicity in patients receiving different PCT regimens was also compared. As shown in Table S1, intergroup differences in acute toxicities showed no statistical significance.

\section{Multivariate analysis}

All 451 eligible patients were included in the multivariate analysis (Table 3). We found that the use of CTX/NTZ was not a significant independent prognostic factor for OS (CTX/NTZ plus PCT vs PCT alone: HR, 0.92; 95\% CI, $0.61-1.40 ; P=0.695)$. Meanwhile, patients with an advanced $\mathrm{N}$ stage exhibited a higher risk of death (N2-3 vs N0-1:
HR, 1.66; 95\% CI, 1.18-2.35; $P=0.004)$. Moreover, patients with multiple metastatic sites were found to exhibit worse OS than patients with bone-only metastasis (HR, 2.73; $95 \%$ CI, 1.94-3.85, $P<0.001)$. In addition, LRRT use was a protective factor for de novo metastatic patients (HR, 0.56; 95\% CI, 0.40-0.77; $P<0.001)$.

\section{Interaction effects}

Furthermore, we examined the interaction effects between the treatment regimen (CTX/NTZ plus PCT vs PCT) and other prognostic factors ( $\mathrm{N}$ stage, LRRT, and metastatic sites) after adjusting for age, gender, $\mathrm{T}$ stage, $\mathrm{N}$ stage, metastatic sites, PCT cycle, LRRT use, and treatment regimen. As shown in Table 4, no significant interaction effects were observed between the treatment regimen (CTX/NTZ plus PCT vs PCT) and N stage (HR, 1.05; 95\% CI, 0.29-3.75; $P=0.946)$. Similarly, LRRT use and metastatic sites did not exhibit any interaction effect with the treatment regimen $(P>0.05)$.

\section{Discussion}

To our knowledge, this is the first study exploring the effectiveness of CTX/NTZ on the basis of palliative chemotherapy in metastatic NPC patients in a large cohort of 451 patients. According to data from our matched cased-control cohort, CTX/NTZ combined with PCT yielded a similar treatment efficacy as compared with PCT alone. Moreover, the incidence rates of G3-4 mucositis and G3 skin reactiosn were higher in the CTX/NTZ plus PCT group. 
Table 2 Acute toxicities in de novo NPC patients in different treatment groups

\begin{tabular}{|c|c|c|c|c|c|c|}
\hline & \multirow{2}{*}{$\begin{array}{l}\text { PCT alone } \\
\mathrm{N}=248\end{array}$} & \multirow{2}{*}{$\begin{array}{l}\text { CTX/NTZ plus PCT } \\
\mathrm{N}=62\end{array}$} & \multirow[t]{2}{*}{ PI value } & \multirow{2}{*}{$\begin{array}{l}\text { CTX plus } \mathrm{PCT} \\
\mathrm{N}=25\end{array}$} & \multirow{2}{*}{$\begin{array}{l}\text { NTZ plus } P C T \\
N=37\end{array}$} & \multirow[t]{2}{*}{ P2 value } \\
\hline & & & & & & \\
\hline \multicolumn{7}{|l|}{ Leukocytopenia } \\
\hline G0-I & $87(35.1 \%)$ & $21(33.9 \%)$ & 0.819 & 7 (28.0\%) & $14(37.8 \%)$ & $0.735 \ddagger$ \\
\hline G2 & $94(37.9 \%)$ & $23(37.1 \%)$ & & $9(36.0 \%)$ & | $4(37.8 \%)$ & \\
\hline G3 & $58(23.4 \%)$ & $14(22.6 \%)$ & & $7(28.0 \%)$ & 7 (I8.9\%) & \\
\hline G4 & $9(3.6 \%)$ & $4(6.5 \%)$ & & $2(8.0 \%)$ & $2(5.4 \%)$ & \\
\hline \multicolumn{7}{|l|}{ Neutropenia } \\
\hline G0-I & $97(39.1 \%)$ & $19(30.6 \%)$ & 0.522 & $6(24.0 \%)$ & $13(35.1 \%)$ & 0.016 \\
\hline $\mathrm{G} 2$ & $57(23.0 \%)$ & $19(30.6 \%)$ & & $7(28.0 \%)$ & $12(32.4 \%)$ & \\
\hline G3 & $55(22.2 \%)$ & $13(21.0 \%)$ & & $3(12.0 \%)$ & $10(27.0 \%)$ & \\
\hline G4 & $39(15.7 \%)$ & $\mathrm{II}(\mathrm{I} 7.7 \%)$ & & $9(36.0 \%)$ & $2(5.4 \%)$ & \\
\hline \multicolumn{7}{|l|}{ Anemia } \\
\hline G0-I & $158(63.7 \%)$ & 37 (59.7\%) & 0.336 & $13(52.0 \%)$ & $24(64.9 \%)$ & $0.203 \ddagger$ \\
\hline G2 & $54(21.8 \%)$ & $16(25.8 \%)$ & & $9(36.0 \%)$ & 7 (I8.9\%) & \\
\hline G3 & 21 (8.5\%) & 8 (12.9\%) & & $2(8.0 \%)$ & $6(16.2 \%)$ & \\
\hline G4 & $15(6.0 \%)$ & I (I.6\%) & & I (4.0\%) & $0(0.0 \%)$ & \\
\hline \multicolumn{7}{|l|}{ Thrombocytopenia } \\
\hline G0-I & $184(74.2 \%)$ & $54(87.1 \%)$ & $0.019 \ddagger$ & $22(88.0 \%)$ & 32 (86.5\%) & $1.000 \ddagger$ \\
\hline G2 & 37 (14.9\%) & $2(3.2 \%)$ & & I (4.0\%) & I (2.7\%) & \\
\hline G3 & 18 (7.3\%) & $2(3.2 \%)$ & & I (4.0\%) & I $(2.7 \%)$ & \\
\hline G4 & $9(3.6 \%)$ & $4(6.5 \%)$ & & I (4.0\%) & $3(8.1 \%)$ & \\
\hline \multicolumn{7}{|l|}{ Vomiting } \\
\hline G0-I & $190(76.6 \%)$ & 42 (67.7\%) & 0.357 & 18 (72.0\%) & 24 (64.9\%) & $0.760 \ddagger$ \\
\hline G2 & 47 (19.0\%) & $16(25.8 \%)$ & & $6(24.0 \%)$ & $10(27.0 \%)$ & \\
\hline G3 & II (4.4\%) & $4(6.5 \%)$ & & I (4.0\%) & $3(8.1 \%)$ & \\
\hline \multicolumn{7}{|l|}{ Skin reaction } \\
\hline G0-I & 179 (72.2\%) & 29 (46.8\%) & $<0.001$ & $7(28.0 \%)$ & 22 (59.5\%) & 0.016 \\
\hline G2 & $52(21.0 \%)$ & $15(24.2 \%)$ & & $6(24.0 \%)$ & 9 (24.3\%) & \\
\hline G3 & 17 (6.9\%) & $18(29.0 \%)$ & & 12 (48.0\%) & $6(16.2 \%)$ & \\
\hline \multicolumn{7}{|l|}{ Mucositis } \\
\hline G0-I & 98 (39.5\%) & $20(32.3 \%)$ & $<0.001$ & $3(12.0 \%)$ & 17 (45.9\%) & $0.003 \ddagger$ \\
\hline $\mathrm{G} 2$ & $123(49.6 \%)$ & $18(29.0 \%)$ & & $6(24.0 \%)$ & $12(32.4 \%)$ & \\
\hline G3 & 27 (10.9\%) & $19(30.6 \%)$ & & $12(48.0 \%)$ & $7(18.9 \%)$ & \\
\hline G4 & $0(0.0 \%)$ & $5(8.1 \%)$ & & $4(16.0 \%)$ & I (2.7\%) & \\
\hline \multicolumn{7}{|l|}{ Hepatotoxicity } \\
\hline G0-I & $220(88.7 \%)$ & 51 (82.3\%) & 0.369 & $20(80.0 \%)$ & 31 (83.8\%) & $0.391 \ddagger$ \\
\hline $\mathrm{G} 2$ & 21 (8.5\%) & 9 (I4.5\%) & & $5(20.0 \%)$ & $4(10.8 \%)$ & \\
\hline G3 & $7(2.8 \%)$ & $2(3.2 \%)$ & & $0(0.0 \%)$ & $2(5.4 \%)$ & \\
\hline \multicolumn{7}{|l|}{ Nephrotoxicity } \\
\hline G0-I & $238(96.0 \%)$ & $60(96.8 \%)$ & 1.000 & $23(92.0 \%)$ & 37 (100.0\%) & $0.159 \ddagger$ \\
\hline G2 & $9(3.6 \%)$ & $2(3.2 \%)$ & & $2(8.0 \%)$ & $0(0.0 \%)$ & \\
\hline G3 & I (0.4\%) & $0(0.0 \%)$ & & $0(0.0 \%)$ & $0(0.0 \%)$ & \\
\hline
\end{tabular}

Notes: The PI value was calculated between the CTX/NTZ plus PCT arm and PCT alone arm; The P2 value was calculated between the CTX plus PCT arm and NTZ plus PCT arm. The $P$-value was calculated with the Pearson $\chi 2$ test or Fisher's exact test ( $¥)$.

Abbreviations: NTZ, nimotuzumab; CTX, cetuximab; PCT, palliative chemotherapy. 
Table 3 Multivariate analyses of variables correlated with the treatment regimen status and other prognostic factors in eligible 45 I patients

\begin{tabular}{|c|c|c|c|}
\hline Characteristic & HR & $95 \% \mathrm{Cl}$ & $P$-value \\
\hline \multicolumn{4}{|l|}{ Gender } \\
\hline Male & Reference & & \\
\hline Female & 0.81 & $0.56-1.17$ & 0.270 \\
\hline \multicolumn{4}{|l|}{ Age (years) } \\
\hline$\leq 46$ & Reference & & \\
\hline$>46$ & 1.14 & $0.88-1.49$ & 0.325 \\
\hline \multicolumn{4}{|l|}{ T stage } \\
\hline TI-2 & Reference & & \\
\hline T3-4 & 0.96 & $0.68-1.37$ & 0.833 \\
\hline \multicolumn{4}{|l|}{$\mathrm{N}$ stage } \\
\hline No-I & Reference & & \\
\hline N2-3 & 1.66 & $1.18-2.35$ & 0.004 \\
\hline \multicolumn{4}{|l|}{ Metastatic site } \\
\hline Bone & Reference & & \\
\hline Lung vs Bone & 0.75 & $0.47-1.20$ & 0.226 \\
\hline Liver vs Bone & 1.27 & $0.81-1.99$ & 0.299 \\
\hline Distant nodes vs Bone & 0.68 & $0.34-1.35$ & 0.270 \\
\hline Multiple vs Bone & 2.73 & $1.94-3.85$ & $<0.001$ \\
\hline \multicolumn{4}{|l|}{ PCT cycle } \\
\hline$\leq 4$ & Reference & & \\
\hline$>4$ & 0.86 & $0.62-1.17$ & 0.335 \\
\hline \multicolumn{4}{|l|}{ LRRT } \\
\hline No & Reference & & \\
\hline Yes & 0.56 & $0.40-0.77$ & $<0.001$ \\
\hline \multicolumn{4}{|l|}{ Treatment regimen } \\
\hline PCT alone & Reference & & \\
\hline CTX/NTZ plus PCT & 0.92 & $0.61-1.40$ & 0.695 \\
\hline
\end{tabular}

Notes: The data are obtained from all $45 \mathrm{I}$ patients included in the study. A Cox proportional hazard model was used to perform multivariate analyses. All variables were transformed into categorical variables.

Abbreviations: HR, hazard ratio; $\mathrm{Cl}$, confidence interval; NTZ, nimotuzumab; CTX, cetuximab; LRRT, locoregional radiotherapy; PCT, palliative chemotherapy.

Approximately $15 \%$ of NPC patients were diagnosed with de novo metastatic cancer, which usually demonstrated a more aggressive nature, before the initiation of any treatment. ${ }^{5}$ The platinum-based PCT was established as the standard treatment for metastatic population with an estimated response rates of $>50 \% .{ }^{6,13-15}$ However, the duration of response and survival time remained limited, while second-line chemotherapy was only effective in patients with good response. ${ }^{16}$ Novel anti-cancer drugs were urgently needed to alleviate the plight. EGFR is highly expressed in human epithelial carcinomas and its expression is reportedly correlated with a more aggressive phenotype accompanied by resistance to treatment and poor prognosis. ${ }^{8}$ The use of monoclonal antibodies of EGFR could help block the aberrant signaling in malignant cells. In fact, several studies have reported the presence of EGFR overexpression in NPC, and offered further evidence for the use of anti-EGFR drugs in NPC treatment. ${ }^{17-22}$

The treatment effect of anti-EGFR drugs has been reported in locally advanced NPC patients. Based on the results of a prospective phase II study conducted by Ma et al, patients who received CCRT and CTX achieved a 2-year progression free survival (PFS) rate of $86.5 \%{ }^{23}$ Similarly, Liu et al evaluated the efficacy and safety of the combination of CCRT and NTZ. In the cohort of 42 patients, NTZ was well tolerated in locoregionally advanced NPC patients with a 2-year distant metastasis free survival (DMFS) and OS of $93.1 \%$ and $96.6 \%$ respectively. ${ }^{24}$ Moreover, You et al conducted a retrospective study based on a matched case-control study with a larger cohort, ${ }^{12}$ and the researchers found that CTX/NTZ plus CCRT could improve the OS, PFS, and DMFS in comparison with CCRT alone, and the toxicity was comparable in these 2 groups. Therefore, we believe that the addition of $\mathrm{CTX} / \mathrm{NTZ}$ to PCT could further improve the OS in metastatic NPC patients.

Thus far, only a few studies have explored the role of anti-EGFR drugs in metastatic NPC. Xu et al reported a single-arm study assessing the efficacy and toxicity of CTZ in the combined treatment of recurrent and/or metastatic NPC patients. ${ }^{25}$ The researchers found that CTX may be effective for the 2-year OS rate reached $53.3 \%$ in this cohort. Moreover, the toxicities resulting from the addition of CTX to the chemoradiotherapy were tolerable. Chan et al performed a multicenter, phase II study of CTX in combination with carboplatin in patients with recurrent or metastatic NPC. The median OS time was 233 days in all patients, and 6 of these patients (10\%) experienced serious treatment-related adverse events. ${ }^{9}$ To our knowledge, no study has compared the survival of metastatic patients with or without anti-EGFR drug treatment.

In the present study, all patients were diagnosed with metastatic NPC prior to receiving any treatment. Using a relatively large cohort and the PSM method, we found that patients treated with CTX/NTZ plus PCT achieved a similar 3-year OS rate as compared to patients with PCT alone (63.0\% vs $58.1 \% ; P=0.485)$. With regard to toxicity, we found that severe mucositis and dermatitis were more common in the CTX subgroup, which was consistent with previous studies. ${ }^{10,12,23}$ One possible reason for this 
Table 4 Interaction between the treatment regimen status and other significant prognostic factors

\begin{tabular}{|c|c|c|c|}
\hline Characteristic & Adjusted HR & $95 \% \mathrm{Cl}$ & $P$-value \\
\hline \multicolumn{4}{|l|}{ Model I } \\
\hline \multicolumn{4}{|l|}{ EGFR-targeted regimen } \\
\hline PCT alone & Reference & & \\
\hline CTX/NTZ plus PCT & 0.89 & $0.27-2.92$ & 0.840 \\
\hline \multicolumn{4}{|l|}{$N$ stage } \\
\hline NI-O & Reference & & \\
\hline N2-3 & 1.66 & $1.16-2.37$ & 0.006 \\
\hline \multicolumn{4}{|l|}{ Interaction effect } \\
\hline CTX/NTZ plus PCT*N2-3 & 1.05 & $0.29-3.75$ & 0.946 \\
\hline \multicolumn{4}{|l|}{ Model 2} \\
\hline \multicolumn{4}{|l|}{ EGFR-targeted regimen } \\
\hline PCT alone & Reference & & \\
\hline CTX/NTZ plus PCT & 0.89 & $0.67-3.28$ & 0.330 \\
\hline \multicolumn{4}{|l|}{ LRRT use } \\
\hline No & Reference & & \\
\hline Yes & 0.59 & $0.42-0.82$ & 0.002 \\
\hline \multicolumn{4}{|l|}{ Interaction effect } \\
\hline CTX/NTZ plus PCT*LRRT & 0.54 & $0.21-1.36$ & 0.190 \\
\hline \multicolumn{4}{|l|}{ Model 3} \\
\hline \multicolumn{4}{|l|}{ EGFR-targeted regimen } \\
\hline PCT alone & Reference & & \\
\hline CTX/NTZ plus PCT & 1.20 & $0.69-2.07$ & 0.523 \\
\hline \multicolumn{4}{|l|}{ Metastatic site } \\
\hline Bone & Reference & & \\
\hline Lung vs Bone & 0.82 & $0.50-1.134$ & 0.424 \\
\hline Liver vs Bone & 1.48 & $0.92-2.37$ & 0.103 \\
\hline Distant nodes vs Bone & 0.73 & $0.35-1.52$ & 0.395 \\
\hline Multiple vs Bone & 2.80 & $1.95-4.01$ & $<0.001$ \\
\hline \multicolumn{4}{|l|}{ Interaction effect } \\
\hline CTX/NTZ plus PCT*Lung & 0.34 & $0.04-2.73$ & 0.311 \\
\hline CTX/NTZ plus PCT*Liver & 0.29 & $0.06-1.35$ & 0.115 \\
\hline CTX/NTZ plus PCT*Distant nodal & 0.62 & $0.07-5.31$ & 0.661 \\
\hline CTX/NTZ plus PCT*Multiple & 0.91 & $0.33-2.51$ & 0.861 \\
\hline
\end{tabular}

Notes: The data are obtained from all $45 \mathrm{I}$ patients included in the study. The multivariable Cox regression model was adjusted for age, gender, $\mathrm{T}$ stage, $\mathrm{N}$ stage, metastatic site, PCT cycle, LRRT use, and treatment regimen.

Abbreviations: HR, hazard ratio; Cl, confidence interval; NTZ, nimotuzumab; CTX, cetuximab; LRRT, locoregional radiotherapy; PCT, palliative chemotherapy.

finding might be that CTX is a chimeric mouse-human IgG1 monoclonal antibody, which displays higher binding affinity to cells expressing high EGFR levels. ${ }^{26}$ Given these negative results, we believe that the use of CTX/ NTZ in de novo metastatic NPC patients should be carefully considered in the clinical setting. Moreover, novel treatment methods such as the introduction of immune checkpoint inhibitors should be explored. Therefore, we have initiated a worldwide, multicenter, phase III study of cisplatin and gemcitabine with or without PD-1 antibody (toripalimab) in patients with recurrent or metastatic NPC, and the results are awaited (NCT 03581786).

The present study has certain limitations. First, this is a retrospective study, and although some selected biases were 
eliminated via propensity scoring, the presence of other confounding factors persisted. Moreover, all the patients involved in this study were enrolled from a single treatment center in a high-prevalence area, and the histological subtype of all patients was World Health Organization type III. Thus, a multicenter prospective study may be needed to better evaluate the role of CTX/NTZ in metastatic NPC patients.

\section{Conclusion}

This study demonstrated that de novo metastatic NPC patients may not benefit from the addition of anti-EGFR drugs, whereas the addition of CTX to CCRT might exacerbate the acute mucositis and skin reactions.

\section{Ethics approval and consent to participate}

This retrospective study was approved by the Clinical Research Committee of Sun Yat Sen University Cancer Center. Patients were required to provide written informed consent before enrolling in the study.

\section{Acknowledgment}

This work was supported by grants from the National Key R\&D Program of China (2016YFC0902003, 2017YFC1 309003, 2017YFC0908500).

\section{Author contributions}

All authors contributed to data analysis, drafting and revising the article, gave final approval of the version to be published, and agree to be accountable for all aspects of the work.

\section{Disclosure}

The authors report no conflicts of interest in this work.

\section{References}

1. Ferlay J, Soerjomataram I, Dikshit R, et al. Cancer incidence and mortality worldwide: sources, methods and major patterns in GLOBOCAN 2012. Int J Cancer. 2015;136:E359-86. doi:10.1002/ ijc. 29210

2. Chua M, Wee J, Hui E, Chan A. Nasopharyngeal carcinoma. Lancet. 2016;387:1012-1024. doi:10.1016/S0140-6736(15)00055-0

3. Wei WI, Sham JS. Nasopharyngeal carcinoma. Lancet. 2005;365:2041-2054. doi:10.1016/S0140-6736(05)66698-6

4. Lo K, To K, Huang D. Focus on nasopharyngeal carcinoma. Cancer Cell. 2004;5:423-428.

5. Tang L, Chen Q, Fan W, et al. Prospective study of tailoring wholebody dual-modality $[18 \mathrm{~F}]$ fluorodeoxyglucose positron emission tomography/computed tomography with plasma Epstein-Barr virus DNA for detecting distant metastasis in endemic nasopharyngeal carcinoma at initial staging. J Clin Oncol. 2013;31:2861-2869. doi:10.1200/ JCO.2012.46.0816
6. Zhang L, Huang Y, Hong S, et al. Gemcitabine plus cisplatin versus fluorouracil plus cisplatin in recurrent or metastatic nasopharyngeal carcinoma: a multicentre, randomised, open-label, phase 3 trial. Lancet. 2016;388:1883-1892. doi:10.1016/S0140-6736(16)31388-5

7. Tsang J, Lee VH, Kwong DL. Novel therapy for nasopharyngeal carcinoma-where are we. Oral Oncol. 2014;50:798-801. doi:10.1016/j.oraloncology.2014.01.002

8. Ciardiello F, Tortora G. A novel approach in the treatment of cancer: targeting the epidermal growth factor receptor. Clin Cancer Res. 2001;7:2958-2970.

9. Chan AT, Hsu MM, Goh BC, et al. Multicenter, phase II study of cetuximab in combination with carboplatin in patients with recurrent or metastatic nasopharyngeal carcinoma. $J$ Clin Oncol. 2005;23:3568-3576. doi:10.1200/JCO.2005.02.147

10. You R, Sun R, Hua YJ, et al. Cetuximab or nimotuzumab plus intensity-modulated radiotherapy versus cisplatin plus intensitymodulated radiotherapy for stage II-IVb nasopharyngeal carcinoma. Int J Cancer. 2017;141:1265-1276. doi:10.1002/ijc.30819

11. Lin M, You R, Liu YP, et al. Beneficial effects of anti-EGFR agents, Cetuximab or Nimotuzumab, in combination with concurrent chemoradiotherapy in advanced nasopharyngeal carcinoma. Oral Oncol. 2018;80:1-8. doi:10.1016/j.oraloncology.2018.03.002

12. You R, Hua YJ, Liu YP, et al. Concurrent chemoradiotherapy with or without Anti-EGFR-targeted treatment for stage II-IVb nasopharyngeal carcinoma: retrospective analysis with a large cohort and long follow-up. Theranostics. 2017;7:2314-2324. doi:10.7150/thno.19710

13. Ji J, Yun T, Kim S, et al. A prospective multicentre phase II study of cisplatin and weekly docetaxel as first-line treatment for recurrent or metastatic nasopharyngeal cancer (KCSG HN07-01). Eur J Cancer. 2012;48:3198-3204. doi:10.1016/j.ejca.2012.06.009

14. Ngan R, Yiu H, Lau W, et al. Combination gemcitabine and cisplatin chemotherapy for metastatic or recurrent nasopharyngeal carcinoma: report of a phase II study. Ann Oncol. 2002;13:1252-1258. doi:10.1093/annonc/mdf200

15. Chen C, Wang F, An X, et al. Triplet combination with paclitaxel, cisplatin and 5-FU is effective in metastatic and/or recurrent nasopharyngeal carcinoma. Cancer Chemother Pharmacol. 2013;71:371378. doi:10.1007/s00280-012-2020-x

16. Chan AT, Teo PM, Leung TW, Johnson PJ. The role of chemotherapy in the management of nasopharyngeal carcinoma. Cancer. 1998;82:10031012. doi:10.1002/(sici)1097-0142(19980315)82:6<1003::aid-cncr1 $>3.0$. co;2-f

17. Zheng X, Hu L, Chen F, Christensson B. Expression of Ki67 antigen, epidermal growth factor receptor and Epstein-Barr virus-encoded latent membrane protein (LMP1) in nasopharyngeal carcinoma. Eur J Cancer B Oral Oncol. 1994;30b:290-295.

18. Roychowdhury DF, Tseng A Jr., Fu KK, et al. New prognostic factors in nasopharyngeal carcinoma. Tumor angiogenesis and C-erbB2 expression. Cancer. 1996;77:1419-1426. doi:10.1002/(SICI)10970142(19960415)77:8<1419::AID-CNCR1 >3.0.CO;2-7

19. Sheen TS, Huang YT, Chang YL, et al. Epstein-Barr virus-encoded latent membrane protein 1 co-expresses with epidermal growth factor receptor in nasopharyngeal carcinoma. Jpn $J$ Cancer Res. 1999;90:1285-1292. doi:10.1111/j.1349-7006.1999.tb00710.x

20. Fujii M, Yamashita T, Ishiguro R, et al. Significance of epidermal growth factor receptor and tumor associated tissue eosinophilia in the prognosis of patients with nasopharyngeal carcinoma. Auris Nasus Larynx. 2002;29:175-181.

21. Leong JL, Loh KS, Putti TC, et al. Epidermal growth factor receptor in undifferentiated carcinoma of the nasopharynx. Laryngoscope. 2004;114:153-157. doi:10.1097/00005537-200401000-00029

22. Putti TC, To KF, Hsu HC, et al. Expression of epidermal growth factor receptor in head and neck cancers correlates with clinical progression: a multicentre immunohistochemical study in the AsiaPacific region. Histopathology. 2002;41:144-151. doi:10.1046/ j.1365-2559.2002.01436.x 
23. Ma BB, Kam MK, Leung SF, et al. A phase II study of concurrent cetuximab-cisplatin and intensity-modulated radiotherapy in locoregionally advanced nasopharyngeal carcinoma. Ann Oncol. 2012;23:1287-1292. doi:10.1093/annonc/mdr401

24. Liu ZG, Zhao Y, Tang J, et al. Nimotuzumab combined with concurrent chemoradiotherapy in locally advanced nasopharyngeal carcinoma: a retrospective analysis. Oncotarget. 2016;7:24429-24435. doi:10.18632/oncotarget.8225
25. Xu T, Ou X, Shen C, Hu C. Cetuximab in combination with chemoradiotherapy in the treatment of recurrent and/or metastatic nasopharyngeal carcinoma. Anticancer Drugs. 2016;27:66-70. doi:10.1097/ CAD.0000000000000294

26. Ramakrishnan MS, Eswaraiah A, Crombet T, et al. Nimotuzumab, a promising therapeutic monoclonal for treatment of tumors of epithelial origin. MAbs. 2009;1:41-48. doi:10.4161/mabs.1.1.7509

\section{Publish your work in this journal}

Drug Design, Development and Therapy is an international, peerreviewed open-access journal that spans the spectrum of drug design and development through to clinical applications. Clinical outcomes, patient safety, and programs for the development and effective, safe, and sustained use of medicines are a feature of the journal, which has also been accepted for indexing on PubMed Central. The manuscrip management system is completely online and includes a very quick and fair peer-review system, which is all easy to use. Visit http://www. dovepress.com/testimonials.php to read real quotes from published authors. 